\title{
SUPPLEMENTO
}

\section{Esperienza di aferesi nelle unità intensive}

\author{
Giorgio Splendiani ${ }^{1}$, Franco Turani ${ }^{2}$, Annalisa Noce ${ }^{3}$, Anna Mudoni ${ }^{4}$, Nicola Di Daniele ${ }^{3}$ \\ ${ }^{1}$ Servizio di Nefrologia e Dialisi, "Aurelia Hospital", Roma \\ ${ }^{2}$ Servizio di Anestesia e Rianimazione, "European Hospital", Roma \\ ${ }^{3}$ Dipartimento di Medicina dei Sistemi - Servizio di Nefrologia e Ipertensione, Università degli studi di Roma "Tor \\ Vergata", Roma \\ ${ }^{4}$ U.O.C. di Nefrologia e Dialisi, Ospedale "Cardinale Panico", Tricase, Lecce
}

\begin{abstract}
EXPERIENCE OF APHERESIS IN THE INTENSIVE CARE UNIT
Abstract. Introduction: Sepsis is one of the main causes of death in critically ill patients worldwide, and in many cases it is associated with renal and/or other organ failure. However, we do not have an efficient therapy to reduce its extremely high mortality rate. In the last years the interest around the use of extracorporeal blood purification techniques has increased. One of the emerging treatments in patients with severe sepsis and septic shock is coupled plasma filtration adsorption (CPFA). CPFA is a novel extracorporeal blood purification therapy aimed at non-selectively reducing the circulating levels and activities of both pro and anti-inflammatory mediators. The aim of this study, conducted in septic patients, was to observe the effects of CPFA on mortality and several laboratory and hemodynamic parameters.

Patients and methods: We treated 65 patients $(50$ males and 15 females) with CPFA. We then analysed the mean arterial pressure (MAP), the sequential organ failure assessment (SOFA) score, and the acute physiology and chronic health evaluation II (APACHE II) score. Serum levels of interleukin-6 and procalcitonin were also measured.
\end{abstract}

Results: After 28 days we observed a mortality of 36.9\% (24 patients) and a significant reduction in IL-6, procalcitonin, SOFA and APACHE scores.

Conclusion: CPFA represents a promising new approach for blood purification in sepsis.

Key words: CPFA, Sepsis, Procalcitonin, Interleukin-6

Conflict of interest: None.

Financial support: None.

Ricevuto: 21 Gennaio 2013; Accettato: 27 Febbraio 2013

\section{Introduzione}

I pazienti ricoverati presso le Unità di Terapia Intensiva richiedono spesso l'uso di trattamenti extracorporei per insufficienza di uno o più organi o per la presenza di affezioni sistemiche, quali la sepsi (1). Le tecniche extracorporee di emodepurazione, che possono essere utilizzate a tale scopo, comprendono: l'emodialisi, la plasmaferesi e l'emoperfusione (2). In particolare, la plasmaferesi, nelle sue varie modalità di applicazione, trova indicazione da sola o associata ad altre tecniche emodepurative $(3,4)$.

Le tecniche di aferesi terapeutica sono: il plasma exchange, la plasmaferesi a cascata e il plasma adsorbimento.

Il plasma exchange è utilizzato prevalentemente nella Sindrome di Goodpasture, nella Sindrome di Guillain-Barré, nella Miastenia e nella Porpora Trombotica Trombocitopenica (5). La plasmaferesi a cascata è utilizzata nel Mieloma, nella Crioglobulinemia, in varie forme di immunopatologie e nel trattamento delle sindrome da iperviscosità (reoferesi) (6).
La plasmaferesi selettiva con uso di colonne adsorbenti (plasma adsorbimento) trova, invece, particolare indicazione, associata all'emodialisi, nella SIRS (Systemic Inflammatory Response Syndrome) e nella MODS (Multiple-Organ-Dysfunction-Syndrome) (7).

La SIRS e lo shock settico rappresentano le cause più frequenti di mortalità e morbidità nelle unità di Terapia Intensiva, determinando 135.000 morti all'anno in Europa e 270.000 morti all'anno negli USA. Il meccanismo fisiopatologico della SIRS è caratterizzato da un'alterazione della permeabilità della barriera intestinale ai germi, con conseguente batteriemia e liberazione di LPS (Lipo-Poli-Saccaridi) da parte della parete batterica dei Gram negativi. I LPS si legano alle proteine plasmatiche con attivazione della cascata infiammatoria, attivazione dei monociti-macrofagi e liberazione, nelle prime fasi di tale processo, di citochine proinfiammatorie (Tumor Necrosis Factor- $\alpha$, Interleuchina-1, Interleuchina-6 e Interleuchina-8) e, successivamente, di citochine antinfiammatorie (Interleuchina-4 e Interleuchina-10). Le citochine, a loro volta, induco- 


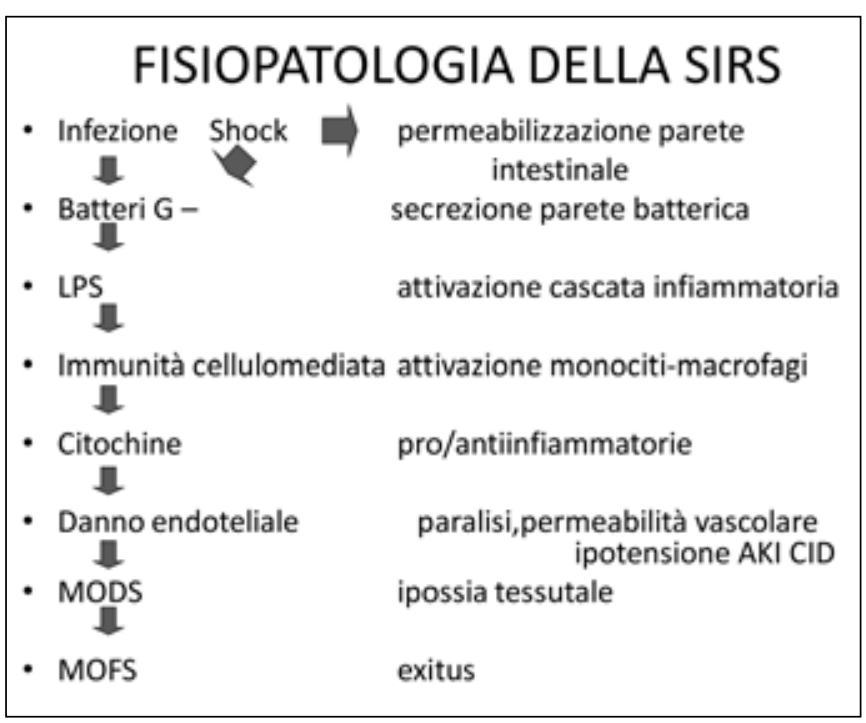

Fig. 1 - Schema del meccanismo fisiopatologico della SIRS.

no danno endoteliale con paralisi e aumento della permeabilità vascolare, ipotensione e danno sia ischemico che ipossico a carico di vari organi (Fig. 1). Le dirette conseguenze di tali processi sono la MODS (Multiple Organ Dysfunction Syndrome) e la MOF (Multisystem-Organ-Failure) (8).

Valutando dettagliatamente le fasi che caratterizzano la SIRS si osserva che in ognuna di esse vi è una specifica indicazione ad effettuare una tecnica extracorporea in base alla funzione da essa svolta. L'emodialisi con filtri particolari (Oxiris) o l'emoperfusione su Polimixina B provocano la riduzione dei LPS e, pertanto, sono indicate nelle prime fasi sella SIRS. La tecnica combinata plasma adsorbimento su resina ed emodialisi denominata CPFA (Coupled Plasma Filtration Adsorbtion) induce, invece, una riduzione delle citochine $(9,10)$.

La CRRT (Continuous Renal Replacement Therapy), la MARS e la DECAP trovano indicazioni rispettivamente nell'insufficienza renale, epatica o respiratoria (11) (Fig. 2).

Scopo del nostro lavoro è stato valutare l'impatto della CPFA, in pazienti settici ricoverati presso il reparto di Terapia Intensiva, sulla mortalità e l'effetto sui parametri ematochimici utilizzati per la diagnosi e il monitoraggio della sepsi.

\section{Pazienti e Metodi}

Tutti i dati dello studio sono stati analizzati in modo anonimo e l'approvazione dello studio è stata ottenuta dal Comitato Etico del Policlinico Universitario di "Tor Vergata".

Lo studio è stato condotto dal 2 Gennaio del 2007 al 30 Agosto del 2012, per un periodo di follow-up complessivo di 5 anni. Sono stati arruolati 65 pazienti ( 50 maschi, 15 femmine), ricoverati presso le unità di terapia intensiva dell'European Hospital, dell'"Aurelia Hospital" e del Policlinico Universitario di "Tor Vergata". Al momento dell'arruolamento nello studio, sono stati raccolti i dati riguardanti età, sesso, peso cor-

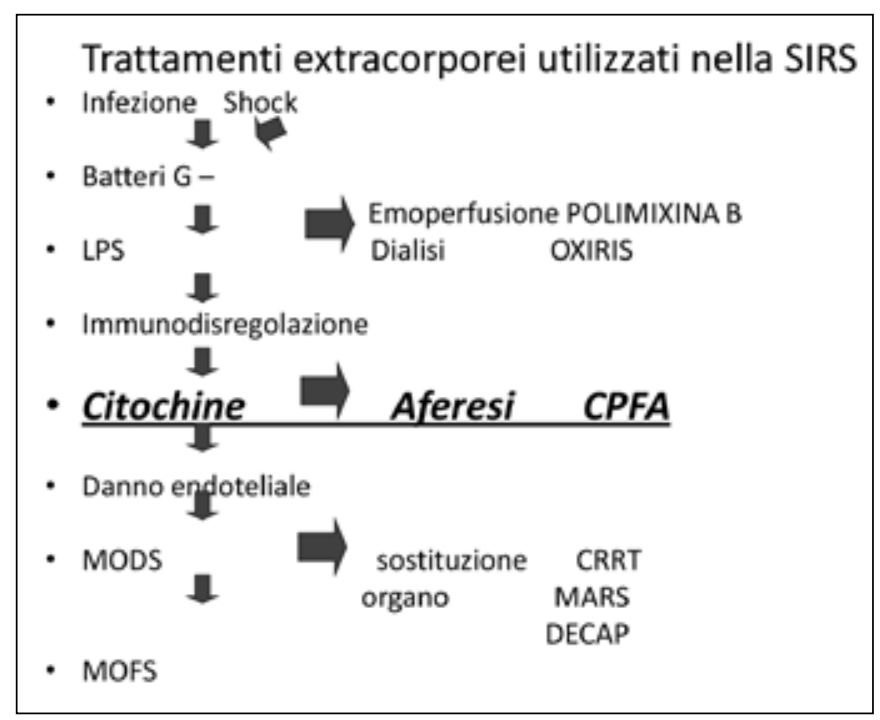

Fig. 2 - Razionale per cui i diversi trattamenti extracorporei vengono utilizzati nelle varie fasi della SIRS.

poreo, data d'ingresso, motivo del ricovero e principali score prognostici.

L'età media dei pazienti era di $65+8$ anni.

Il SOFA score medio calcolato nei soggetti arruolati era $15 \pm 2$ e l'APACHE II (Acute Physiology and Chronic Health Evaluation) presentava un valore medio di $24 \pm 6$ al momento dell'arruolamento.

L'infusione di noradrenalina media è stata pari a $0.35 \pm 0.8$ $\mathrm{micr} / \mathrm{Kg} / \mathrm{min}$ a partire dal momento dell'arruolamento e fino alla sospensione.

Mediamente, i pazienti presentavano l'insufficienza di $3 \pm 1$ organi.

La CPFA è stata eseguita per un tempo medio di $8.1 \mathrm{~h}+1.5$ $\mathrm{h}$, utilizzando monitor LYNDA (Bellco, Mirandola, Italy), un emofiltro costituito da una membrana a elevata biocompatibilità in polieteresulfone, di superficie $1.20 \mathrm{~m}^{2}$, sterilizzato con raggi gamma (DIAPE S-HF ${ }^{\circledR}$ Bellco, Mirandola, Italy), un plasmafiltro di $0.45 \mathrm{~m}^{2}$, sterilizzato con raggi gamma, costituito da una membrana a elevata biocompatibilità in polieteresulfone (MICROPE $\mathrm{S}^{\circledR}$ Bellco, Mirandola, Italy) e da una cartuccia sorbente contenente $70 \mathrm{~g}$ di resina stirenica (stirene divinil-benzene) ad alta biocompatibilità, con una superficie di $700 \mathrm{~m}^{2} / \mathrm{g}$ con diametro interno dei pori di 30 nanometri, sterilizzata a calore umido (MediaSorb ${ }^{\circledR}$, Bellco, Mirandola, Italy). I valori di flusso ematico erano compresi tra i 200 e i $250 \mathrm{~mL} / \mathrm{min}$ e la quantità di soluzione di reinfusione utilizzata era di $35 \mathrm{~mL} / \mathrm{kg} / \mathrm{h}$. Il valore del flusso plasmatico (Qp) era pari al $15 \%$ rispetto al flusso ematico prescritto. L'accesso vascolare utilizzato è stato un catetere temporaneo a doppio lume in vena femorale (57 pazienti) o catetere temporaneo a doppio lume in vena giugulare interna destra ( 9 pazienti).

La media dei trattamenti a cui era sottoposto ogni paziente era pari a $4.12+1.2$.

La concentrazione plasmatica di IL-6 è stata determinata in 
duplicato, utilizzano un Kit immunoenzimatico ad alta sensibilità (Mactech AB, Nacka, Sweden). Per la determinazione della procalcitonina (PCT) è stato utilizzato un metodo automatico VIDAS ${ }^{\circledR}$ B.R.A.H.M.S. (bio Mérieux, Marcy L'Etoile, France). Il limite più basso di determinazione era di $0.05 \mathrm{ng} / \mathrm{mL}$, mentre la sensibilità di tale metodica era pari a $0.09 \mathrm{ng} / \mathrm{mL}$.

I pazienti arruolati sono stati sottoposti a un prelievo ematico al momento dell'arruolamento per la determinazione di procalcitonina e IL-6. Prelievi successivi sono stati effettuati ogni 24 ore.

\section{Analisi statistica}

I dati sono stati espressi come media \pm deviazione standard. Per valutare la distribuzione del campione è stato utilizzato il test di Kolmogorov-Smirnov. Per l'analisi delle variabili sono stati utilizzati il T-Test per dati appaiati e il test di Wilcoxon, mediante il software SPSS11.01 Software (SPSS forWindows, Rel. 11.0.1. 2001, SPSS Inc., Chicago, IL). Un valore di $\mathrm{p}<0.05$ era considerato statisticamente significativo.

\section{Risultati}

Nei 65 pazienti oggetto dello studio si è osservata una riduzione statisticamente significativa dei valori di calcitonina $(24.5 \pm 6.8$ $\mathrm{pg} / \mathrm{mL}$ vs $9.3 \pm 3.5 \mathrm{pg} / \mathrm{mL}, \mathrm{p}<0.001)$ e di IL-6 $(352 \pm 320 \mathrm{pg} / \mathrm{mL}$ vs $137 \pm 140 \mathrm{pg} / \mathrm{mL}, \mathrm{p}<0.05)$ rispetto ai valori basali.

Inoltre, la somministrazione di noradrenalina si è ridotta significativamente alla fine del trattamento con CPFA rispetto al momento dell'inizio dell'infusione, prima della CPFA $(0.8$ $\mathrm{micr} / \mathrm{Kg} / \mathrm{min}$ vs $0.4 \mathrm{micr} / \mathrm{Kg} / \mathrm{min}, \mathrm{p}<0.001)$.

La pressione arteriosa media, monitorizzata in continuo, è aumentata in maniera significativa da $62 \pm 5$ a $73 \pm 2, \mathrm{p}<0.05)$.

Inoltre, abbiamo osservato una diminuzione significativa degli score SOFA (da $15 \pm 2$ a $12 \pm 3, \mathrm{p}<0.05$ ) e APACHE II (da $24 \pm 6$ a $21 \pm 5, \mathrm{p}<0.05$ ) ed è migliorato il rapporto $\mathrm{PaO}_{2} / \mathrm{FIO}$ $(162 \pm 83.3$ vs $244 \pm 130, \mathrm{p}<0.001)$.

La mortalità a 28 giorni dal ricovero in Terapia Intensiva nei pazienti trattati con CPFA è stata di 24 pazienti, pari al $36.9 \%$.

\section{Discussione}

Dall'analisi dei risultati emerge che la mortalità al $28^{\circ}$ giorno risulta del $36.9 \%$, a fronte di una predetta probabilità media di morte intraospedaliera del $75 \%$, stimata secondo il sistema di classificazione SAPS II. Inoltre, la percentuale di mortalità per sepsi osservata risulta inferiore rispetto a quella da noi osservata in un precedente lavoro che era pari al $51 \%$ dei pazienti trattati (12) e rispetto a quella riportata dal GiViTi, che era pari al $65.3 \%$ (13). Si sono osservati anche un significativo miglioramento del SOFA score (da $15 \pm 2$ a $12 \pm 3$ ), indice del danno multiorgano, e un miglioramento significativo di alcuni parametri emodinamici sistemici come la pressione arteriosa media (da $62 \pm 5$ a $73 \pm 2, \mathrm{p}<0.05$ ), con conseguente riduzione progressiva della terapia con ammine vasoattive.

Inoltre, è stato constatato un incremento del rapporto tra pressione arteriosa dell' $\mathrm{O}_{2}$ e la frazione di ossigeno inspirata $\mathrm{PaO}_{2} /$ $\mathrm{FIO}_{2}(162 \pm 83.3$ vs $244 \pm 130, \mathrm{p}<0.001)$, indice della funzione respiratoria.
Si è rilevato un significativo miglioramento dei valori di procalcitonina, parametro che viene utilizzato per la diagnosi e il monitoraggio della sepsi, e dell'IL-6, la cui riduzione a valori inferiore a $400 \mathrm{pg} / \mathrm{mL}$ può essere considerata un segno prognostico favorevole.

I dati provenienti da questo studio, pertanto, sembrano incoraggianti poiché mostrano un importante trend di miglioramento di molte delle variabili prese in esame.

\section{Conclusioni}

La plasmaferesi rimane una tecnica utile nelle Terapie Intensive e nei pazienti critici.

Il plasma adsorbimento si è dimostrato efficace e utile nella riduzione delle citochine nella sepsi; inoltre, la CPFA sembrerebbe migliorare l'emodinamica e la prognosi nei pazienti con SIRS e MODS. In conclusione, si può sperare che un'ulteriore evoluzione tecnologica e un maggiore uso di metodi di depurazione extracorporea possano indurre una sempre maggiore riduzione della morbilità e della mortalità dei pazienti con shock settico.

\section{Riassunto}

Introduzione. La sepsi è una delle principali cause di morte in tutto il mondo nei pazienti ricoverati in Terapia Intensiva e, in molti casi, è associata a insufficienza renale e/o di un altro organo. Tuttavia, non esiste una terapia efficace per ridurre questo tasso di mortalità estremamente elevato. Negli ultimi anni, l'interesse intorno all'utilizzo di tecniche extracorporee di emopurificazione è aumentato. Uno dei trattamenti emergenti nei pazienti con sepsi grave e shock settico è la CPFA, una nuova terapia extracorporea di emopurificazione finalizzata a una riduzione non selettiva dei livelli circolanti e delle attività dei mediatori sia proinfiammatori che antinfiammatori.

Lo scopo di questo studio è stato di osservare gli effetti della CPFA nei pazienti settici sulla mortalità e su alcuni parametri di laboratorio ed emodinamici.

Pazienti e Metodi. Abbiamo trattato con CPFA 65 pazienti (50 maschi e 15 femmine). La pressione arteriosa media (MAP), il SOFA score e I'APACHE II score sono stati monitorati. Sono stati dosati i livelli sierici di Interleuchina-6 e di procalcitonina.

Risultati. Abbiamo osservato una mortalità di 24 pazienti $(36.9 \%)$ a 28 giorni e una significativa riduzione di IL-6, di procalcitonina e dei SOFA e APACHE II score.

Conclusioni. La CPFA rappresenta una nuova e promettente terapia da utilizzare nei pazienti con sepsi.

Parole chiave: CPFA, Sepsi, Procalcitonina, Interleuchina-6

Dichiarazione di conflitto di interessi: Gli Autori dichiarano di non avere conflitto di interessi.

Contributi economici degli autori: Gli Autori dichiarano di non aver ricevuto sponsorizzazioni economiche per la preparazione dell'articolo. 
Indirizzo degli Autori:

Prof. Giorgio Splendiani

Servizio di Nefrologia e Dialisi

"Aurelia Hospital"

Via Aurelia 860

00133 Roma

splendianigiorgio@libero.it

\section{Bibliografia}

1. Ronco C, Bellomo R. Acute renal failure and multiple organ dysfunction in the ICU from renal replacement therapy (RRT) to multiple organ support therapy (MOST). Int J Artif Organs 2002; 25: 733-47.

2. Nalesso F. Trattamento extracorporeo dell'insufficienza reale acuta (AKI) durante la Sepsi. G Ital Nefrol 2012; 29 (Suppl. 56): 535-40.

3. Tetta C. L'aferesi in rianimazione in L'aferesi terapeutica, Editoriale Bios 2002; 105-10.

4. Ronco C. Terapie extracorporee speciali in L'aferesi terapeutica, Editoriale Bios 2005; 23-32.
5. Splendiani G, Stuart S. Tecniche depurative nelle patologie extrarenali in La dialisi Tecnica e clinica, S.E.U. 2007; 106-15.

6. Ferrannini M, Vischini G, Staffolani E, et al. Rheopheresis in vascular diseases. Int J Artif Organs 2007; 30 (10): 923-9.

7. Bosch T. Recent advances in therapeutic apheresis. J Artif Organs 2003; 6 (1): 1-8.

8. Cohen J. The immunopathogenesis of sepsis. Nature 2002; 420 (6917): 885-91.

9. Mokart D., Merlin M, Sannini A, et al. Procalcitonin, interleukin 6 and systemic inflammatory response syndrome (SIRS):early markers of postoperative sepsis after major surgery. Br J Anaest 2005; 94 (6): 767-73.

10. Bellomo R, Honoré PM, Matson J, Ronco C, Winchester J. Extracorporeal blood treatment (EBT) methods in SIRS/Sepsis. Int J Artif Organs 2005; 28 (5): 450-8.

11. Bellomo R, Tetta C, Ronco C. Coupled plasma filtration adsorption. Intensive Care Med 2003; 29 (8): 1222-8.

12. Turani F, Falco M, Barchetta R, et al. Different effect of CVVHDF and coupled plasma filtration and adsorption on IL-6 and procalcitonin in sepsis. Critical Care 2011;15 (Suppl 1): S42.

13. GiViTI. Le infezioni in terapia intensiva. Rapporto del progetto di sorveglianza del GiViTI, anno 2005. Ricerca e Pratica 2007; 23: 148-59. 